\title{
Desafíos de la Cardiología: desde la investigación básico-clínica a la era digital
}

\author{
Edgardo Escobar \\ FACC, FAHA \\ Profesor de Medicina. Universidad de Chile. Maestro de la Cardiología Chilena \\ Director Médico, ITMS, Telemedicina de Chile. \\ Conferencia Zapata Díaz, Conferencia Inaugural del LIV Congreso Chileno de Cardiología y Cirugía Cardiovascular. \\ Santiago de Chile 30 de noviembre, 2017
}

Este artículo resume algunos de los trabajos realizados por el autor en las áreas de las Ciencias Básicas y Clínicas, seguidos por lo que es su trabajo actual en Telemedicina. Se describe lo que es la Telemedicina como herramienta fundamental en la atención médica actual, tanto para el informe de exámenes, como en atención médica a distancia, con énfasis en al campo de la Cardiología. Se da una visión de lo que será la práctica médica en el futuro con la aplicación de herramientas, como el Internet de las cosas, la inteligencia artificial, Big Data y la robótica, que se traducirán en una práctica médica cada vez más tecnológica y con menor contacto directo entre pacientes y médicos. Se insiste en que pese al progreso que significa la aplicación de tales herramientas, lo más importante sigue siendo la Prevención de la Enfermedades cardiovasculares, con énfasis en nuestra población infantil (prevención primordial). 


\section{Introducción:}

Es para el que habla un honor el haber sido distinguido con esta Conferencia que honra la memoria del Dr. Jorge Zapata Díaz.

Después de tantos años de trayectoria en nuestra especialidad, decidí hacer un resumen de lo que ha sido mi recorrido en la investigación en las áreas de las ciencias básicas y clínicas, de lo que es mi actividad presente y, lo que creo más importante, de dar una visión de lo que puede ser la práctica médica en el futuro, especialmente pensando en las nuevas generaciones.

Desde mis inicios estuve interesado en la investigación, porque siempre estuve convencido de que es la base de una buena docencia y de una buena práctica médica ${ }^{1}$. Lo que relataré a continuación no incluye todas las investigaciones en que he participado como autor principal o coautor, sino sólo las que me parecen más relevantes y que han significado, algunas de ellas, contribuciones originales.

\section{Pasado}

Mi primera participación en investigación básica estuvo relacionada con la Ateroesclerosis, estudiando en la rata los mecanismos que pudieran estar involucrados en el hecho de que estos animales no la presentan espontáneamente. ${ }^{2}$

En USA participé en numerosos estudios de función ventricular en el animal de experimentación, específicamente en el perro ${ }^{3-5}$, así como en el ser humano ${ }^{6,7}$. Sólo quiero destacar en la anemia normovolémica la importante participación del sistema simpático en la respuesta cardiovascular hiperdinámica, con aumento de la función sistólica del ventrículo izquierdo, sólo parcialmente bloqueada por betabloqueadores y, por otro lado, la importancia de la disminución de la viscosidad sanguínea en la menor resistencia vascular sistémica ${ }^{8-10}$.

En Chile, demostramos que el suero de pacientes anémicos crónicos en contacto con músculo papilar aislado de gato tenía un efecto inotrópico positivo a diferencia de los pacientes controles, efecto no modificado por betabloqueo del simpático e independiente de catecolaminas, ya que la concentración plasmática de éstas era la misma en ambos grupos. ${ }^{11}$

Estudios realizados en pericardio aislado y con sobrecarga aguda de volumen en perros con pericardio intacto y pericardiectomizados confirmaron la importancia de la rigidez del pericardio como mecanismo protector de sobredistensión de las cavidades cardiacas ${ }^{12,13}$.

En el perro intacto observamos que la pared ventricular sufre un engrosamiento del $16,6 \%$ al final de la sístole, lo que tiene importancia para los cálculos de tensión o "stress"14.

A continuación, quiero mencionar 3 estudios sobre Prostaglandinas (PGs).

El primero fue la demostración de PGs E-2 y F2 alfa en biopsias de tejido auricular de pacientes sometidos a cirugía cardiaca ${ }^{15}$. El segundo, demostró el aumento significativo de dichas PGs en plasma venoso de pacientes con insuficiencia aórtica o insuficiencia mitral asintomáticas, comparados a un grupo control ${ }^{16}$, hallazgos que en conjunto nos permiten sugerir que las prostaglandinas a través de sus efectos vasodilatadores y/o inotrópicos positivos pueden jugar un rol en la respuesta cardiovascular en estos casos.

El tercero demostró que la razón PG E2 /tromboxano en muestras de seno coronario de pacientes, disminuye significativamente durante la isquemia inducida por "pacing", disminución que es neutralizada por la administración previa de Captopril, sugiriendo la utilidad que puede tener su administración en cuadros coronarios agudos. ${ }^{17}$ En un ámbito clínico, hicimos una serie de estudios en Miocardiopatías, y quiero señalar la frecuencia del Toxoplasma Gondii como causa de miocardiopatía dilatada, demostrando su presencia en el miocardio en la autopsia de uno de estos pacientes. ${ }^{18}$

En el campo de la cardiopatía coronaria efectuamos una serie de trabajos, especialmente con técnicas radioisotópicas. Destacaría a este respecto el análisis de la relación entre Hipertensión y Cardiopatía coronaria ${ }^{19}$ y haber sido uno de los revisores de la tercera definición universal del Infarto del Miocardio. ${ }^{20}$

La función ventricular en pacientes ha sido de nuestro particular interés.

En el hipertiroidismo, al igual que en la anemia, la respuesta hiperdinámica y el aumento de la función ventricular sistólica son sólo parcialmente bloqueadas por la administración de betabloqueadores, por lo que se deduce un efecto inotrópico positivo directo de la hormona tiroidea $^{21}$.

En el área de las valvulopatías demostramos, con técnicas radioisotópicas, que en la insuficiencia mitral crónica asintomática no sólo puede existir una disminución de la fracción de eyección del ventrículo izquierdo, sino también del ventrículo derecho, en ausencia de hipertensión pulmonar en reposo, factor que a nuestro juicio debe ser considerado en la decisión quirúrgica en estos pacientes. ${ }^{22}$

En la Estenosis aórtica estudiamos la variabilidad de la frecuencia cardiaca en su dominio tiempo y frecuencia antes y después de la cirugía valvular. Precirugía existe 
una significativa disminución de la actividad parasimpática con predominio de la actividad simpática, similar a los pacientes de alto riesgo de arritmias ventriculares malignas post infarto agudo del miocardio, alteración que se normaliza en controles un año después de la cirugía. ${ }^{23}$

En Insuficiencia aórtica aguda estudiamos sus características clínicas y seguimiento así como los resultados del tratamiento quirúrgico, perentorio en estos pacientes apenas las condiciones clínicas así lo permitan. ${ }^{24}$

En insuficiencia aórtica crónica hemos realizado numerosas investigaciones en una cohorte de 140 casos: características clínicas, electrocardiográficas y ecocardiográficas, relación de la capacidad funcional con parámetros de función ventricular obtenidos en forma invasiva, su historia natural, valor pronóstico de los índices de función ventricular, etc. Las conclusiones principales son las actualmente muy bien conocidas: disminución de la fracción de eyección y de índices inotrópicos (velocidad de acortamiento circunferencial, razón de presión de fin sístole/volumen de fin sístole) aun en pacientes asintomáticos; mala evolución y deficiente resultado postoperatorio sobrepasados ciertos límites de dilatación al final del diástole y del sístole; uso de vasodilatadores arteriales e inhibidores de la enzima convertidora para disminuir el grado de dilatación del ventrículo izquierdo.

Es importante enfatizar, como está actualmente bien establecido, la necesidad de cirugía en pacientes asintomáticos con insuficiencia aórtica, así como mencionamos para la insuficiencia mitral, cuando se detectan precozmente alteraciones de la función ventricular. Lo anterior está resumido en un artículo de revisión ${ }^{25}$.

\section{Presente}

Hace algunos años tuve la oportunidad de involucrarme con la Telemedicina, TM.

La TM se puede definir como el diagnóstico y tratamiento usando tecnologías de información y comunicaciones (TICS) y tiene dos escenarios básicos: a) sistemas de médico a médico (conectividad entre los proveedores de cuidado médico); b) sistemas de médico a paciente (conectividad entre doctor y paciente, manejo remoto del paciente, telemonitoreo, educación y terapia).

Convencido de que era una forma de atención médica especialmente útil en Chile como apoyo para la atención primaria, por la insuficiente cantidad de especialistas y las dificultades de acceso a la atención médica dada nuestra extensa ruralidad, abandoné otras responsabilidades y asumí la Dirección médica de un proyecto de Tele-electrocardiografía. Por las razones señaladas, hasta ese momento existía una gran demora en la citación y en el informe de los electrocardiogramas y un gran número de informes realizados por no especialistas.

El sistema que utilizamos permite la adquisición en pocos minutos del electrocardiograma (ECG) de 12 derivaciones, igual que el ECG tradicional, con un equipo de muy pequeño tamaño, y su trasmisión por teléfono móvil o fijo, o por internet o desde un computador. Cualquiera sea la señal de trasmisión, una plataforma, diseñada por ingenieros chilenos, (PIT, Plataforma Integrada de Telemedicina), la transforma en una señal digital que se expresa en la imagen del ECG (Fig. 1). Esta es recibida en un Centro Telemédico donde un paramédico se asegura que está técnicamente correcta y que no es igual a algún trazado que pudiera haber quedado almacenado en el equipo, que interroga sobre síntomas y datos biodemográficos y que cuando todos estos pasos están cumplidos, lo que también es de corta duración, la transfiere al computador del médico. El médico informa el ECG en muy pocos minutos gracias a la PIT que permite hacer las mediciones tradicionales del ECG, y "pinchar" los diagnósticos sobre un listado preestablecido en forma muy rápida, de manera que el proceso total no toma más de 5 minutos y el informe es enviado al lugar de origen (Figs $2,3,4)$. Si se estima necesario, el trazado actual puede, además, ser comparado, derivación por derivación, con trazados previos, ya que todos los diagnósticos pasan a una base de datos de donde se pueden recuperar en cualquier momento.

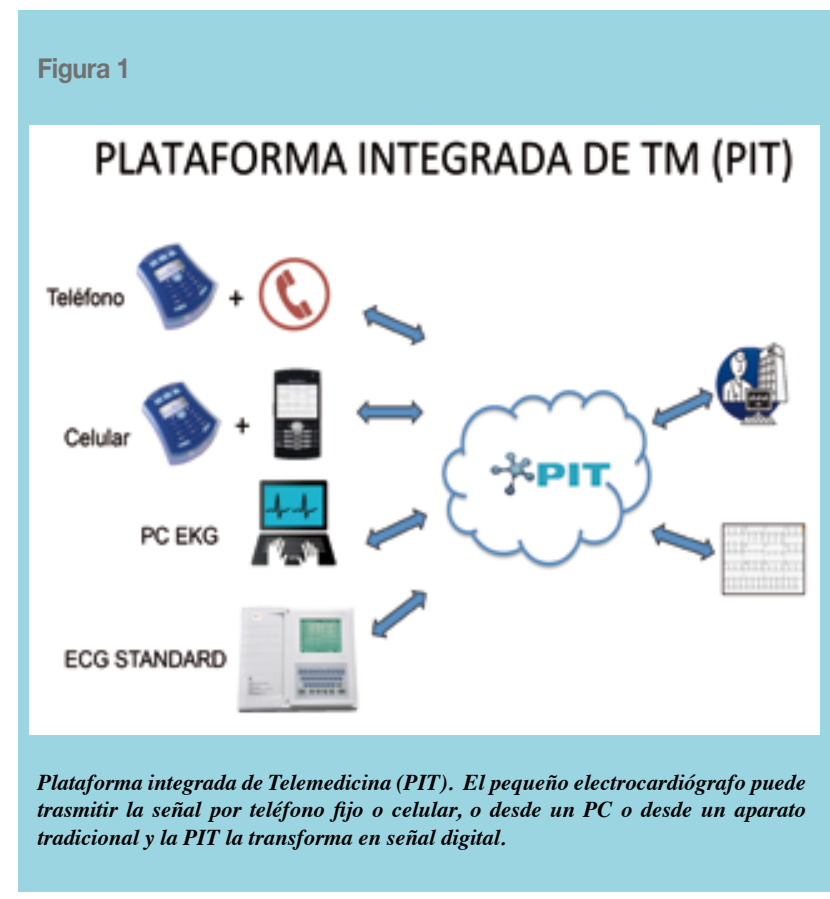




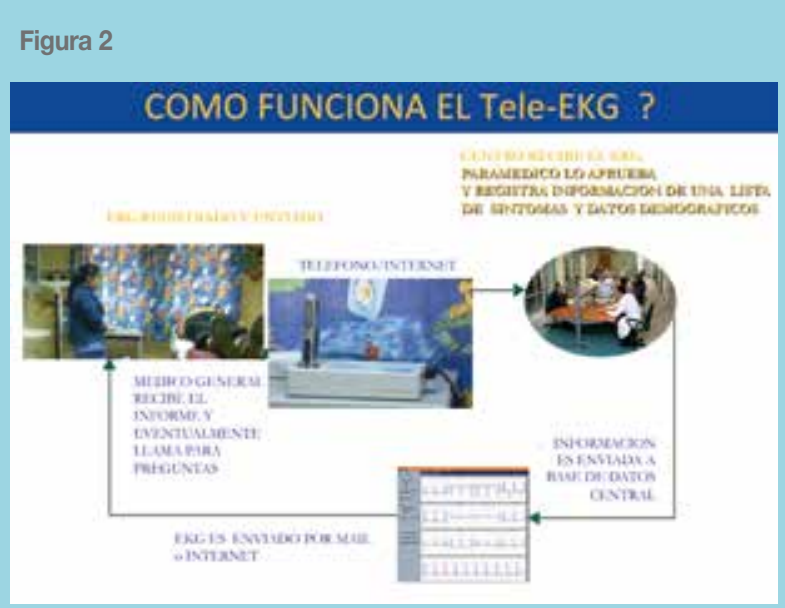

El ECG es trasmitido desde un Policlínico o Sala de urgencia al Centro telemédico donde un paramédico lo recibe y procesa, antes de pasar al computador del médico quien lo informa, volviendo el examen informado a su sitio de origen, por internet of fax.

\section{Figura 3}

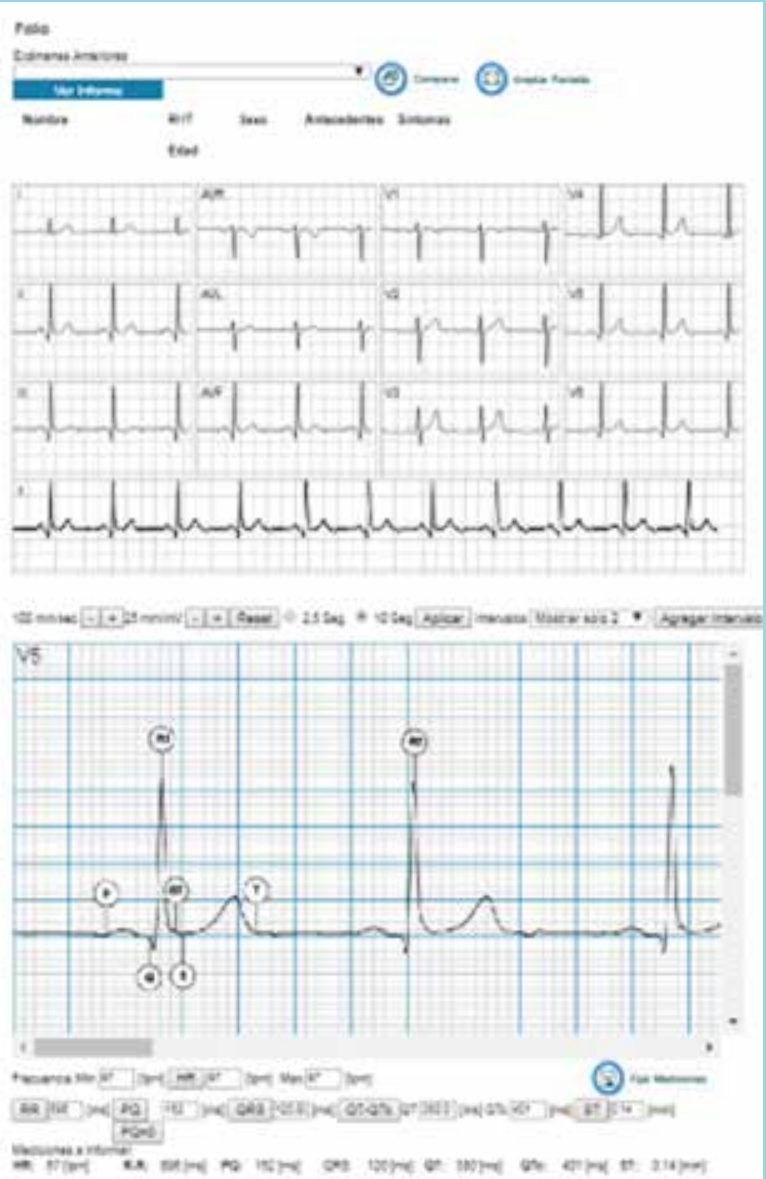

Pantalla que muestra la forma en que el especialista ve el ECG, pudiendo elegir cualquiera derivación para amplificarla y posicionar los vectores para las mediciones habituales.

\section{Figura 4}

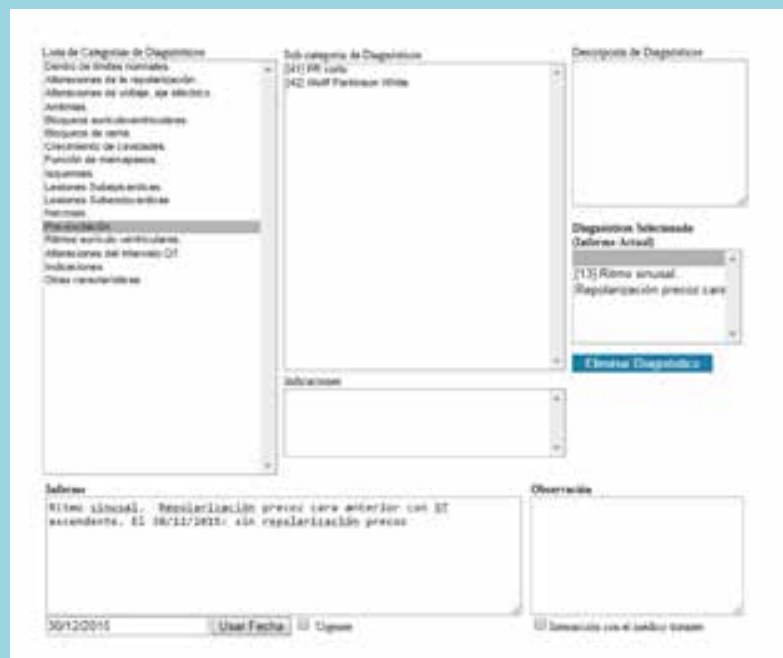

Ejemplo de un listado de diagnósticos, sobre el cual el especialista hace un click para que pasen a la base de datos y al informe final.

Actualmente, los equipos están distribuidos en 650 puntos, 500 dependientes del Ministerio de Salud. Los informes son emitidos en menos de $10 \mathrm{~min}$ si así es requerido (particularmente importante para el diagnóstico prehospitalario del infarto agudo del miocardio). Los trazados son absolutamente iguales a los adquiridos con el equipo tradicional y en el momento actual se reciben más de 50000 ECG al mes y hay más de 7 millones almacenados en nuestra base de datos. Muy importante es enfatizar que se realiza Auditoría de todos los informes y el porcentaje de errores, la enorme mayoría menores, no supera el $1 \%$ del total. ${ }^{26}$

El sistema nos ha permitido estudiar la prevalencia del patrón de Brugada en 122.000 trazados, incluyendo niños. El tipo 1 de Brugada estuvo presente en el 4.6 de cada 10.000 trazados, cifra similar a lo observado en países del hemisferio occidental ${ }^{27}$. Hemos estudiado la frecuencia del intervalo QTc prolongado en diferentes patrones electrocardiográficos en 136.000 trazados, siendo importante destacar que trazados normales pueden mostrar QTc prolongado en el $16 \%$ de los casos y en el $40 \%$ de trazados con hipertrofia ventricular izquierda por voltaje y alteraciones de la repolarización. No todos los bloqueos completos de rama tienen QTc prolongado: sólo el $50 \%$ de los bloqueos completos de rama izquierda y el $25 \%$ de los bloqueos completos de rama derecha ${ }^{28}$. Tuvimos la oportunidad de evaluar el impacto del terremoto de Febrero del año 2010 en la incidencia de lesiones subepicárdicas. Analizamos más de 280.000 ECG registrados en los fines de semana en un periodo de 12 meses 
entre el 2009 y 2010. Hubo un aumento estadísticamente significativo de lesiones subepicárdicas durante el fin de semana del terremoto comparado con cualquier otro fin de semana, siendo más frecuente en mujeres (75\%) lo opuesto a lo observado en condiciones habituales ${ }^{29}$. El estudio de las lesiones subepicárdicas (IAM) en un total de 523.371 ECG, confirmó mayor incidencia de IAM en hombre $(69.2 \%)$, su presentación en mujeres de mayor edad, su mayor frecuencia entre abril y junio, y entre la séptima y novena regiones.

Lo más importante es que se demostró que la TM es una herramienta de gran valor para el diagnóstico precoz del IAM y su tratamiento, particularmente en áreas remotas, e hizo posible la aplicación de la ley AUGE. ${ }^{30}$

La adquisición del ECG prehospitalario en el IAM es de extrema importancia, ya que es el factor de mayor impacto en la probabilidad de recibir reperfusión. La implementación de sistemas bien organizados de cuidado prehospitalario del IAM y el diagnóstico precoz, ayudarán al objetivo de salvar más vidas y prevenir incapacidades innecesarias. ${ }^{31}$

Metha $\mathrm{S}$ et $\mathrm{al}^{32}$, han confirmado la importancia de nuestro sistema de TM en el diagnóstico y acceso a la reperfusión en 3 países latinoamericanos: Brasil, Colombia y México, en un sistema en red con referencia desde consultorios periféricos a un centro único. Son 224 centros y 401.095 pacientes ingresados, diagnosticándose 4.394 IAM con un $95 \%$ de seguridad diagnóstica y utilizando la estrategia fármaco invasiva, con una mortalidad de $4.8 \%$. El sistema en red y el uso de la Telemedicina son fundamentales para el tratamiento oportuno y mejorar la sobrevida, y el Ministerio de Salud está haciendo los esfuerzos por implementarlo.

Además de la electrocardiografía, contamos en nuestro servicio con el informe por Telemedicina de Monitoreo ambulatorio de presión arterial, Monitoreo ambulatorio del ECG de 24 horas, Espirometría e Imagenología. ${ }^{26}$

La TM permite la trasmisión de cualquier tipo de información, incluso a través de dispositivos móviles: signos vitales, biomarcadores, señales de dispositivos implantables, utilizando el Smartphone. Ya existe la posibilidad de enviar un ECG simplemente apoyando los dedos en un dispositivo móvil (Fig. 5) lo que es de gran utilidad para detectar fenómenos agudos y episodios de arritmia. Este sistema ha demostrado su enorme utilidad para detectar episodios de fibrilación auricular. ${ }^{33}$

Podemos hablar en el presente del smartphone "medicalizado" que puede almacenar y/o trasmitir la información arriba señalada, como además la cara y voz del paciente,

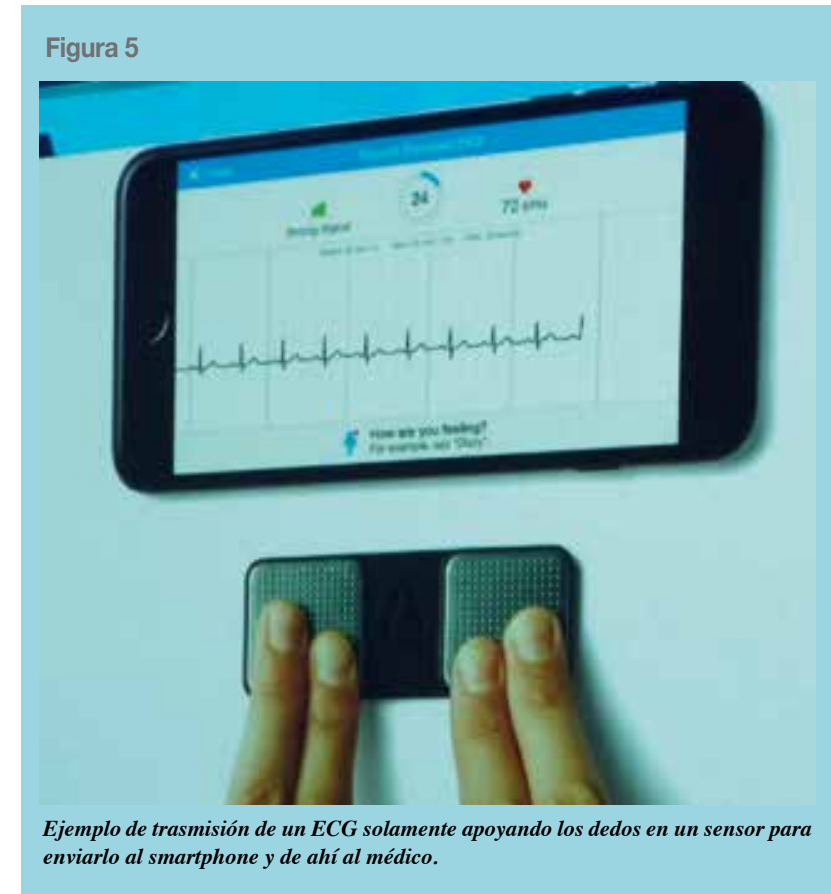

exámenes de laboratorio, imágenes, incluyendo ecocardiogramas, etc. Ya existe la posibilidad de trasmitir un ecocardiograma a través de un celular. Ejemplos concretos del monitoreo a distancia, con la información necesaria son los casos de insuficiencia cardiaca ${ }^{34}$, y control y tratamiento de pacientes diabéticos ${ }^{35}$. Existe desde hace poco un inyector reusable de insulina combinado con una aplicación de Smartphone que ayuda a los diabéticos insulinodependientes a calcular sus dosis y mantener un registro de las horas de inyección. Permite a los pacientes calcular las horas de comida y corregir dosis como también mostrar recordatorios ${ }^{36}$. Dispositivos usables incluyen bandas en las muñecas, parches o bandas torácicas que trasmiten los datos en forma inalámbrica vía Bluetooth o por cable a un Smartphone. Sensores que se insertan en un smartphone incluyen termómetros, monitores de presión arterial, monitores de glicemia, etc. Hay 1.000 compañías fabricando dispositivos que redefinirán el estándar de la atención médica. Ya existe "mi teledoctor" el cual se puede consultar en cualquier momento. ¿Desaparecerá el fonendoscopio? Es posible que sea reemplazado por un miniecocardiógrafo que ya existe, pero cuya aplicación actual está limitada por su costo.

En resumen, la TM está transformando la asistencia sanitaria y social. Es un medio de proporcionar atención médica en áreas remotas por un pequeño número de prestadores de salud para impactar una gran región geográfica. La Telecardiología tiene un gran potencial para reducir la variabilidad de diagnósticos, como también mejorar el tratamiento y 
oferta de servicios de salud aumentando la calidad, eficiencia y costo/efectividad. La Telecardiología puede ayudar a comunidades con servicios escasos porque se sobrepone a las barreras de distancia y tiempo entre los pacientes y prestadores de salud. La implementación de Telecardiología puede tener importantes beneficios socioeconómicos para pacientes, familias y sistemas de salud.

\section{Futuro}

Comparto la opinión de Siegel S et al, que en el congreso de la American Heart Association recién realizado (Nov, 2017) afirmaron que en cuanto a Tecnología y Cuidado de la salud el futuro es brillante.

Hay tres tecnologías que tendrán una influencia muy importante: el Internet de las cosas (IoT por sus siglas en inglés), la Inteligencia artificial (IA) y los Big Data (BD). IoT es la capacidad de transferir datos a través de la red sin requerir interacción de humano a humano o humano a computadora. Desde el uso invasivo de teléfonos inteligentes y miniaturización de casi cualquier cosa a un dispositivo aún más inteligente conectado al ambiente, capturando y alimentando datos en el cuidado médico, el IoT muy probablemente tendrá un impacto en el acceso, calidad y costo del cuidado médico. Representa una gran mejoría en la conectividad.

La IA es un programa de computación diseñado para realizar determinadas operaciones que se consideran propias de la inteligencia humana. Recordemos que en 1996 el programa informático IBM Deep Blue derrotó al campeón mundial de ajedrez. Las predicciones de la IA son múltiples. Por nombrar sólo algunas: el año 2022, transcribir con precisión cualquier audio de lenguaje humano; el 2024 traducir con precisión cualquier idioma; el 2031 gestionar toda la cadena de ventas en una tienda; 2045 las computadoras serán mucho más potentes que todos los cerebros humanos de la tierra juntos; el 2053 operar sin asistencia en un pabellón de cirugía. Se generarán robots autónomos capaces de atender nuestras necesidades. ${ }^{37,38}$ La IA permite automatizar el ingreso a las fichas electrónicas, incluso a través del reconocimiento de la voz del paciente liberando al clínico de la necesidad de enfrentarlo. Se pueden mejorar los diagnósticos y afinar los diagnósticos diferenciales entre otras ventajas. ${ }^{39}$

La IA ya llegó al celular, nuevo Mate 10 Pro de Huawei, cuyo procesador Kiron 970, permite, por ejemplo, la traducción simultánea de textos en imágenes de 50 idiomas distintos. Los científicos del Instituto tecnológico de Massachussets (MIT) crearon un robot que es capaz de generar historias de terror, después de leer 140.000 cuen- tos de terror publicados en un foro de internet.

BD permite almacenar y analizar enormes volúmenes de información ${ }^{40,41}$. Estos sistemas permiten almacenar todos los datos del paciente en un sólo lugar y los hacen disponibles para los médicos que traten a ese paciente. Combinando esta información con múltiples otras, se pueden llegar a predecir cardiopatías sobre la base de mediciones, formas de comportamiento (sueño, dieta, actividad) e historia médica personal y familiar. Podemos predecir cuándo una cardiopatía, como insuficiencia cardiaca, cardiopatía coronaria, hipertensión, empeorarán antes que se necesite hospitalización, y aun antes que el paciente perciba que algo anda mal. Podemos mirar los hábitos diarios en tiempo real para determinar cómo afectan nuestra salud cardiovascular. Podemos determinar qué causa los episodios de fibrilación auricular, cómo interactúan comportamientos, dieta, otras enfermedades, genes para causarla y podemos diseñar tratamientos.

El impacto de todas estas tecnologías ahora y en el futuro, aquí muy resumido, es evidente. La atención médica se alejará cada vez más del hospital y de las oficinas de los médicos. Los hospitales se descentralizarán y el cuidado se centralizará en la Comunidad, para la Prevención, Medicina de urgencia y Cuidado crónico.

Los sistemas de información son herramientas claves: trazabilidad de acciones clínicas, estandarización de procedimientos, continuidad en el cuidado del paciente con historia clínica compartida, servicios en línea, velando por la seguridad de los datos. Crece cada vez más la oferta de asistencia médica por videoconferencia, aplicación disponible para dispositivos con conexión a internet y con staff de facultativos disponibles 24 horas.

Un computador puede leer 100.000 tomografías computadas de corazón en 2 minutos, ecocardiogramas en 1 min y pruebas de esfuerzo en 30 segundos, alimentado por un sistema de entrenamiento de millones de imágenes semejantes.

Hacia el 2025 las visitas virtuales sobrepasarán a las visitas físicas. Encuestas recientes muestran que el $70 \%$ de los usuarios considerarían una atención virtual. El mayor impacto de la atención médica en los próximos cinco años será el del seguimiento y monitoreo de los pacientes, vía aplicaciones del Smartphone. Existirá probablemente el "Dr. Uber", cuya imagen se verá en el celular, se leerá su perfil y el tiempo que demoraría en responder la consulta o llegar al domicilio del paciente. También habrá quioscos médicos en los supermercados o grandes tiendas. Un asistente médico escoltará al consumidor a una cabina privada para una "visita" médica a distancia. 


\section{Prevención}

Si bien los avances tecnológicos son de la mayor importancia, no debemos olvidar que en la base de la práctica cardiológica debe estar la prevención.

En diferentes publicaciones hemos abordado el problema de la prevención cardiovascular en América latina ${ }^{42-46}$. Es importante recordar en que, si bien lo básico y fundamental en prevención primaria son los hábitos de vida saludable, es necesaria en muchas ocasiones la utilización de medicamentos para reforzarla con el uso de fármacos como la aspirina, hipotensores e hipolipemiantes, especialmente estatinas. En casos específicos como la obesidad y el tabaquismo, hay fármacos que pueden contribuir a su control. ${ }^{47}$

Uno de los problemas más serios en prevención es la adherencia a los medicamentos y la tecnología en este caso galénica ha venido en nuestra ayuda. Es el caso de la polipíldora que ha logrado combinar en una misma cápsula tres drogas: aspirina, una estatina y un inhibidor de la enzima convertidora de angiotensina (Fig.6), la administración de la polipíldora ha logrado aumentar en forma significativa la adherencia en comparación al uso de los tres medicamentos por separado, disminuyendo la incidencia de eventos cardiovasculares. Esto es especialmente importante en prevención secundaria y en casos de prevención primaria en pacientes de alto riesgo, como lo han establecido diferentes sociedades científicas internacionales y la Sociedad Chilena de Cardiología. ${ }^{48}$

\section{Figura 6}

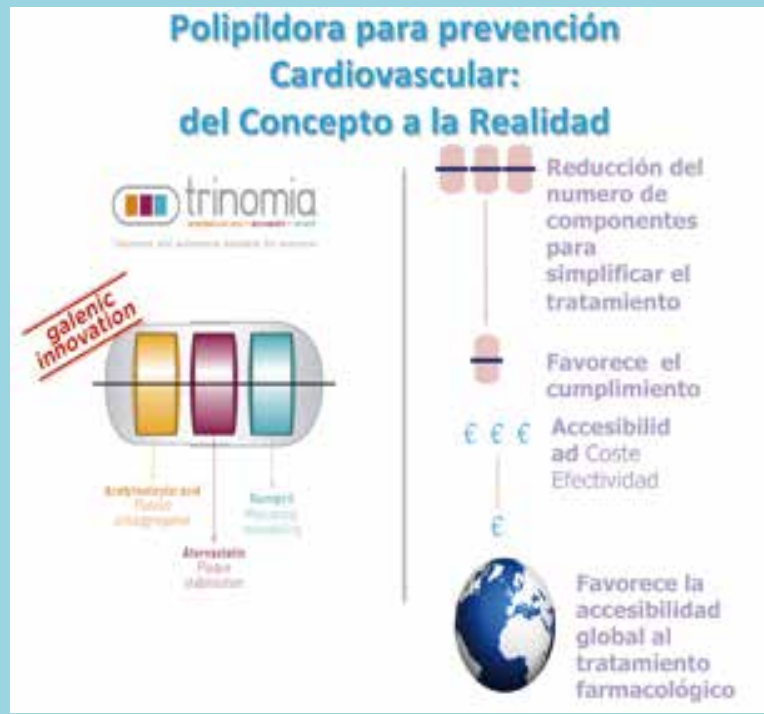

Polipíldora, que contiene los tres componente básicos para prevención secundaria y primaria en casos de alto riesgo: aspirina, estatina, $e$ inhibidor de la enzima convertidora.

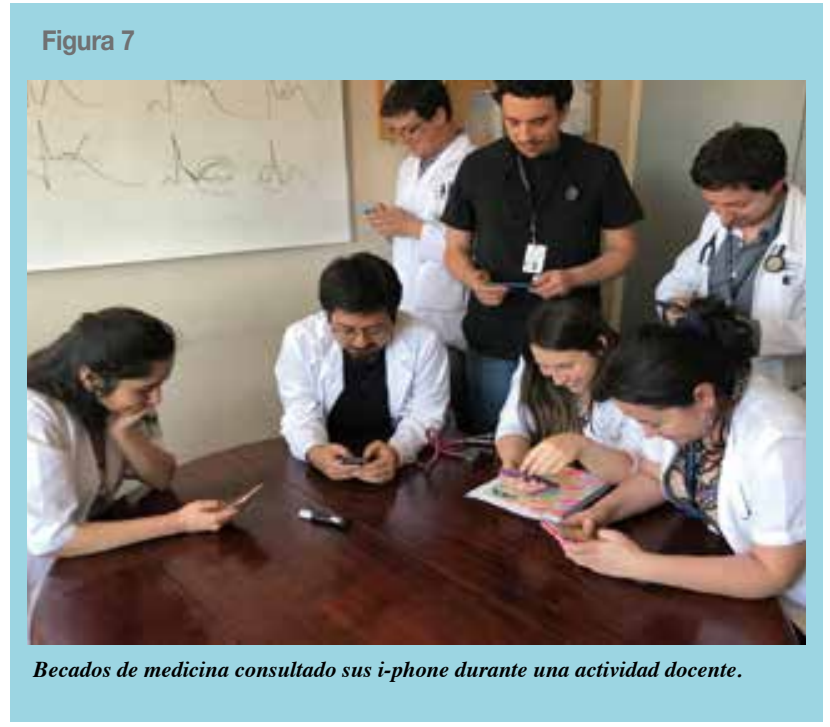

Pero la tecnología ha diseñado otras alternativas para mejorar la adherencia, como son por ejemplo, los envases "inteligentes" que detectan en forma inalámbrica cuando son abiertos, envases que emiten señales auditivas o luminosas como recordatorios del uso de medicamentos, señales que miden cual es el contenido de píldoras o de líquido que queda en el envase, implantación de microchips digeribles en cada píldora, que activados por el jugo gástrico emiten una señal a un parche sensor y de ahí al smartphone. Esta última tecnología ha sido recientemente aprobada por la FDA para el uso de medicamentos en enfermedades psiquiátricas.

En Chile ha habido una positiva experiencia con recordatorios vía mensajes de texto en el tratamiento de la hipertensión. ${ }^{49}$

\section{Conclusiones}

Ha habido cuatro revoluciones industriales: la primera (1784): producción mecánica, vías férreas, poder a vapor; la segunda (1870): producción en masa, poder eléctrico y ensamblajes en línea; la tercera (1969): producción automática, electrónica y computadores; y la cuarta (actual): IoT, IA, Bigdata, robótica. La Medicina está entre la tercera y la cuarta.

El médico actual debe usar cada vez más la tecnología como lo hacen nuestros alumnos durante las reuniones docentes para verificar aspectos específicos y obviamente para almacenar información (Fig. 7). Big data está posicionada para transformar la medicina (Fig. 8)

Chile necesita de profesionales dedicados a la tecnología y no hay suficientes. Los expertos coinciden en que las mallas curriculares están desactualizadas. Habrá un 
Figura 8

\section{Big data está posicionada para transformar la medicina!}

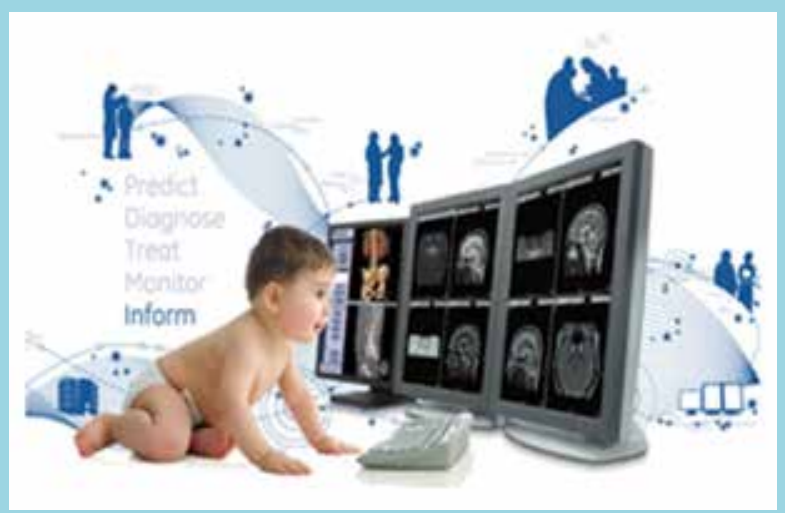

Big data transformará la práctica médica.

déficit de profesionales, específicamente, en las áreas de ciberseguridad, cloud, IoT, Big data y tecnologías de video. Nuestro país debe innovar y avanzar hacia la interoperabilidad y a alcanzar un "Chile digital".

Los médicos somos reacios a adoptar nuevas tecnologías, pero los jóvenes deben estar preparados para adoptarlas para este futuro de la atención médica. Las Facultades de Medicina tienen la principal responsabilidad, pero las Sociedades Científicas pueden contribuir poderosamente. La transformación digital no es únicamente incorporar tecnologías de información a los procesos de la organización para hacerlos más eficientes. Significa, ante todo, un cambio de visión estratégica de la organización que debe comprender rápidamente las tendencias para adaptarse a tiempo a los cambios que le propone el entorno

Hay que evitar hasta donde sea posible que se cumpla la predicción de Einstein que aseveró: "temo el día en que la tecnología sobrepase la interacción humana; el mundo sólo tendrá una generación de idiotas", sino que "hay que estar preparados para el momento en que la IA alcance un nivel en el que será una nueva forma de vida que superará o podría reemplazar a los humanos por completo", como lo dice Stephen Hawking.

Por mi lado quiero enfatizar que ojalá evitemos o disminuyamos la deshumanización de la práctica médica. Los pacientes siempre necesitarán el toque humano del médico.

Un grupo de pacientes al ser preguntados sobre qué debieran saber los médicos, especialmente los jóvenes: que golpeen la puerta antes de entrar a la habitación, que se despidan al salir, que cuando nos hablen nos miren a los ojos. Ninguno pidió la curación de su enfermedad; solo querían RESPETO.

Y nunca debemos olvidar que lo más importante siempre será poner el bienestar de nuestros niños sobre cualquiera otra consideración: Prevención Primordial.

\section{Agradecimientos:}

A mis maestros: Hernán Alessandri, Miguel Hermosilla, Jaime Talesnik, Elliot Rapaport y Julius Comroe. A quienes colaboraron a que gran parte de lo expuesto fuera posible: Gastón Chamorro, Fernando Florenzano, Jorge Jalil, Eduardo Guarda (QEPD), Polentzi Uriarte, Patricio Venegas, Margarita Vejar, Fernando Lanas, José Luis Vukasovic, Patricia Adriazola, Paola Varleta, Roberto Douglas, Berta Zamorano, José Canessa, Milton Alcaíno , Pedro Verdugo, etc, y a todos los integrantes de ITMS, Telemedicina. A mis pacientes y a mis alumnos, a la $\mathrm{SO}-$ CHICAR y en forma especial, a mi Familia. 


\section{Referencias}

1.- ESCOBAR E . Asistencia, Docencia e Investigación Vida Médica 1975; 27:10-12.

2.- DOUGLAS R, ESCOBAR E, ROSENKRANZ A. Rol de las células cebadas en la fisiopatología de la pared arterial. Rev. Med de Chile 1963; 91:193-202.

3.- WONG M, ESCOBAR E, MARTÍNEZ G, RAPAPORT E. Effect of coronary embolization in ventricular volumes . Circulation Res 1965; 16:518-526.

4.- O'ROURKE RA, FISCHER DP, ESCOBAR E, BISHOP VS, RAPAPORT E. ,Effects of acute pericardial tamponade on coronary blood flow. Am J Physiol 1967; 212:549-552.

5.- MURRAY JF, ESCOBAR E, JONES NL, RAPAPORT E. Hemodynamic effects of two beta adrenergic blocking drugs in anesthetized intact dogs. Am Heart J 1966;72:38-49.

6.- RAPAPORT E, WONG M, ESCOBAR E, MARTINEZ G. The effect of upright posture on right ventricular volumes in patients with and without heart failure Am Heart J 1966;11:146-152.

7.- WONG M, ESCOBAR E, MARTINEZ G, RAPAPORT E. The effect of continuous pressure breathing on right ventricular volumes . J Appl.Physiol 1967; 22: 1053-1060.

8.- ESCOBAR E, JONES NL, RAPAPORT E, MURRAY J.F. Ventricular performance in acute normovolemic anemia and effect of beta blockade Am J Physiol 1966; 11:877-884.

9.- MURRAY J.F , ESCOBAR E. Circulatory effects of changes in blood viscosity: comparison of methemoglobinemia and anemia J Appl Physiol 1968; 25: 594-599.

10.-MURRAY J.F, ESCOBAR E, RAPAPORT E. Effects of blood viscosity on hemodynamic response in acute normovolemic anemia Am J Physiol 1969; 216: 638-642.

11. -FLORENZANO F, DIAZ G, REGONESI C, ESCOBAR E. Left ventricular function in chronic anemia: evidence of noncathecolamine positive inotropic factor in the serum. Am J Cardiol 1984; 54: 638-645.

12.- VERDUGO P, MARTINOYA C, ESCOBAR E. Dynamic tensile properties of pericardium, Biophysical mechanics ( Abstract IW 10), 1970.

13. ESCOBAR E. Respuesta ventricular a distintos tipos de sobrecarga. Rev Med de Chile 1976; 104: 554-563.
14.- ESCOBAR E, VERDUGO P, MONTEVERDE L. Tensión sistólica final ventricular izquierda en el perro intacto. Rev.Med. de Chile 1972; 100: 932-936.

15.- ESCOBAR E, ZAMORANO B, GAZMURI R . Demonstration of prostaglandins E-2 and $F 2$ alfa in atrial tissue of patients with heart disease. Am J Cardiol 1983; 52: 424.

16.- ESCOBAR E, ZAMORANO B, GUARDA E, URIARTE P. Concentración de prostaglandinas en plasma venoso en pacientes con valvulopatías. Rev Latina de Cardiología 1988; 9 : 211-218

17.- VEJAR M, ESCOBAR E, VENEGAS P, HERNANDEZ MV, ZAMORANO B. Efectos del Captopril en la relación prostaglandina/tromboxano durante la isquemia inducida por estimulación ventricular.Rev Latina de Cardiología 1994; 15: 149-152

18.- ARRIBADA A, ESCOBAR E. Cardiomyopathy produced by toxoplasma gondii. Am Heart J 1969; 76: 329-339.

19.- ESCOBAR E. Hypertension and coronary artery disease. J Hum Hypertension 2002; 1: S1 61-63.

20.- THYGESEN K, ALPERT JS, JAFFE AS, SIMOONS ML, CHAITMAN BR, WHITE HD. Third Universal definition of Myocardial Infarction. Circulation 2012; 126: 2020-20135.

21.- ESCOBAR E, BIANCHI C, CLAURE H, BOBADILLA E. Volumenes ventriculares izquierdos en el hipertiroidismo Rev Med de Chile 1974; 102: 7-10.

22.- ESCOBAR E, CANESSA J, SIMONS E, VEJAR M. Función ventricular izquierda y derecha en la insuficiencia mitral crónica. Rev.Latina de Cardiologia. 1991; 12:167-173

23.- VUKASOVIC JL, FLORENZANO F, ADRIAZOLA P, ESCOBAR E. Heart rate variability in severe aortic stenosis. J Heart Valve Disease 1999; 8: 143-148.

24.- ESCOBAR E, FLORENZANO F. Insuficiencia aórtica aguda: caracteristicas y seguimiento. Rev Med de Chile 1982; 110: 550-554.

25-. ESCOBAR E. Insuficiencia aórtica "revisitada" Rev Chilena de Cardiología 2007;26: 205-212.

26.- ESCOBAR E, AKEL C. Telemedicine: its importance in Cardiology practice. Experience in Chile.Cardiovasc.Innovations and Applications 2017; 2: 325-331. 
27.- ESCOBAR E, ADRIAZOLA P, BELLO F, ORELLANA M, TREJO P. Prevalence of EKG Brugada pattern ; experience in Chile.Telemed e Health 2008; 14: 64.

28.- ORELLANA M, BELLO F, ESCOBAR E, ADRIAZOLA P, TREJO P, GONZÁLEZ P. Prevalencia del intervalo QT prolongado en diferentes patrones electrocardiográficos. Estudio por Teleelectrocardiografía. Rev Chilena Cardiol 2009; 28;349-356.

29.- BELLO F, ADRIAZOLA P, ESCOBAR E, PAVLOV AS, MEZZANO G, LAMA D. Terremoto 2010: impacto en la incidencia de lesiones subepicárdicas. Experiencia con Telemedicina. Rev Chilena Cardiol 2012;31:189-193.

30.- ESCOBAR E, VEJAR M, DEL PINO R. Lesiones subepicárdicas en Chile (infarto agudo del miocardio con supradesnivel del segmento ST) Experiencia con telemedicina. Rev Chilena Cardiol 2009; 28:73-80.

31.- ESCOBAR E, BARBAGELATA A. Prehospital management of acute STEMI Cardiotext Publishing, LLC Minneapolis, Minn 2015 .ISBN 978-1-935395-66-9.

32.- METHA S, FERRE R, BOTELHO R, FERNANDEZ F, VEGA $\mathrm{R}$, et al. Telemedicince + STEMI systems of care: novel solution to massively expand access to AMI care in developing countries. European Congress of Cardiology, Barcelona, 2017. Abstract 3408.

33.-.KROVOSHEI L, WEBER S, BURKARD T, et al Smart detection of atrial fibrillation 2017 Europace doc 10.1093/euw125.

34.-. Kumar S, Abowd G, Abrhart W et al Center of excellence for mobile sensor data to knowledge J Am Med Ass 2015; 6: 1137-1142

35.-. GOTÉS J. Los avances tecnológicos para el cuidado de los pacientes con diabetes.

https://espanol.medscape.com/vararticulo/5902007_print

36. DAVENPORT L. Smartphone-linked insulin Pen System for Diabetes launched in US. Medscape Dec 18,2017

37. GRACE K. When will Artificial intelligence exceed human performance? Evidence from AI experts. Future of Humanity Institute, 2016

38. TOPOL E. The patient will see you now. Basic Books, Philadelphia 2015ISBN 978-0-465-04054-4
39.- VERGHESE A, SHAH N H, HARRINGTON RA. What this computer needs is a physician. Doi:10.1001/JAMA 2017.19198

40.- \$ Big data en health care. Opportunities and challenges 2015; 3: 209

41.-. RUMFELD JS, JOYNT KE, MADDOX M. Big data analytics to improve cardiovascular care :promises and challenges. Nature Reviews Cardiology 2016; 6: 350-359.

42.-. ESCOBAR E Prevención de las enfermedades cardiovasculares y protección cardiovascular: una perspectiva latinoamericana Cardiology in Review 1996; suppl 1, Dic.

43.- ESCOBAR E. Practical approaches to preventive cardiology. An international symposium. Clinical Cardiology 1997; 20: suppl.(Coeditor).

44.-SCHARGRODSKY H, ESCOBAR MC, ESCOBAR E. Cardiovascular disease prevention. A challenge for Latin America. Circulation 1998; 98: 2103-2104

45.- BUNOUT D, ESCOBAR E. Prevención de las enfermedades cardiovasculares. ¿Deben aplicarse los mismos criterios en América Latina que en Europa y Norteamérica? Rev.Española Cardiol. 2000; 53: 889-895

46.- SANTOS RA, LORENZATTI A, FERNÁNDEZ-BARROS C, ESCOBAR E. Have the results of recent clinical trials of lipid lowering therapies influence the way we should practice? A Latinamaerican perspective of current issues in clinical lipidology. J.Clin.Lipidology 2011;5: 124-132.

47.-ESCOBAR E, VARLETA P. Farmacología en Prevención Primaria ¿son necesarios los medicamentos? Capítulo en libro Prevención cardiovascular. Desafíos y oportunidades. Esper R, Vilariño J.O. editores-Intermédica Editorial. Buenos Aires 2015.ISBN 978-950-43-2744-8.

48.- VEJAR M, ABUFHELE A, VARLETA P, ARAYA MA, ESCOBAR E, FERNANDEZ M, ET AL. Adherencia farmacológica y Prevención secundaria cardiovascular: una de las principales barreras en el tratamiento de la enfermedad ateroesclerótica. Posición del Departamento de Prevención Cardiovascular de SOCHICAR en el uso de la polipíldora en prevención secundaria. Rev Chilena de Cardiol 2016;35:270-282.

49.- VARLETA P, ACEVEDO M, AKEL C, SALINAS C ,NAVARRETE C, GARCIA A, et al. Mobile phone text messaging improves antihypertensive drug adherence in the community. J.Clin Hypertens 2017; 19:1276-1284. 